\title{
Pseudoepiphora from cerebrospinal fluid leak: case report
}

\author{
ROBERT M DRYDEN,' AND ALLAN E WULC \\ From the 'Department of Ophthalmology, University of Arizona, Tucson, Arizona, and \\ ${ }^{2}$ University of Pennsylvania Scheie Eye Institute, Department of Ophthalmology, Philadelphia, \\ Pennsylvania, USA
}

SUMMARY A 4-year-old tearing child with obstruction of the nasolacrimal duct was treated with dacryocystorhinostomy three years after naso-orbital injury. However, what appeared to be tearing peristed, and meningitis developed. Coronal CT scans demonstrated traumatic encephalocele of the posterior superior orbital roof. A chronic orbital cerebrospinal fluid (CSF) leak was diagnosed. To our knowledge no case of chronic CSF leak has been reported that simulated tearing in an otherwise asymptomatic child. In the tearing patient who has a naso-orbital fracture the possibility of chronic CSF leak should be considered.

Lacrimal outflow obstruction is a well recognised complication of midfacial fractures involving the maxilla, the lacrimal bones, or the ethmoids. ${ }^{1-8}$ Common manifestations of obstruction to the lacrimal outflow system include epiphora, dacryocystitis, or mucocele.

A case of epiphora thought to be secondary to nasolacrimal duct obstruction following midfacial trauma is herein described. A 4-year-old child seen two years after naso-orbital injury had traumatic lacrimal duct obstruction and underwent uncomplicated dacryocystorhinostomy (DCR).

The aetiology of tearing in this child was chronic post-traumatic cerebrospinal fluid (CSF) leakage that occurred simultaneously with lacrimal duct obstruction. Despite a successful, DCR, chronic copious CSF leakage simulating tearing continued. To our knowledge this is the first reported case of pseudoepiphora caused by chronic CSF leakage in an otherwise asymptomatic child.

After midfacial fracture chronic CSF leak should be considered in the differential diagnosis of tearing, and the appropriate laboratory and radiographic tests should be performed.

\section{Case report}

A 4-year-old boy was seen with epiphora of the right eye of two years' duration. A motor-car accident two Correspondence to Robert M Dryden, MD, $601 \mathrm{~N}$ Wilmot Road, Suite 58, Tucson, Arizona 85711, USA. years previously resulted in hospital admission, where lethargy progressing to coma and blood in the middle ear led to the diagnosis of basilar skull fracture. Computerised axial tomography (CT) was done three times while he was in hospital and the scans were reported as normal.

Immediately following hospital discharge persistent right-sided tearing was noted. There was no irrigation or pain, and tearing was not related to eating or salivation. Ocular examination showed visual acuities of 20/30 in both eyes. Right inferior oblique overaction was noted with a V-pattern exotropia. Palpation of the orbit revealed no anterior defects and a normally positioned, non-pulsatile globe with no rim defects.

Lacrimal examination with a Jones primary dye test showed no passage of dye after 40 minutes. Traumatic lacrimal drainage obstruction was inferred.

An examination under anaesthesia revealed an obstructed nasolacrimal duct. No fluid could be irrigated through either the upper or lower canaliculus into the nose. Probing revealed a tight internal common punctum and an obstructed nasolacrimal duct.

A dacryocystorhinostomy with canalicular intubation with silicone tubing was performed. The bone was thickened, presumably from past naso-orbital fracture. The patient received $500 \mathrm{mg}$ of intravenous cephazolin (Kefzol) and oral cephalosporins were continued for seven days. 
On the fifth postoperative day mild erythema of the scar site was noted. Tearing had not ceased.

One week after operation the patient developed fever with headache and vomiting. He was admitted to a community hospital with a presumptive diagnosis of periorbital cellulitis and begun on intravenous cephazolin. Two days later he complained of neck pain. A lumbar puncture was done which showed cloudy CSF, raising the possibility of bacterial meningitis. An additional antibiotic was instituted. An axial CT scan was performed which showed air and soft tissue swelling of the right orbit consistent with the operative appearances as well as an opacity of the right ethmoids consistent with the postoperative haemorrhage he had had.

In the ensuing two weeks the patient developed recurrent spiking fevers accompanied by meningeal signs. Intravenous antibiotics were altered to methicillin and chloramphenicol, and he remained afebrile until three weeks after operation, at which time another temperature spike to 101 degrees was noted. The patient was transferred to Tucson Medical Center for further evaluation.

On admission, the temperature was $38 \cdot 3^{\circ} \mathrm{C}$ and the vital signs were otherwise normal. No periorbital cellulitis was noted. The dacryocystorhinostomy scar was healing well. The silicone stent was in place. Tearing was persistent and the results of the eye examination were otherwise normal. The medical examination, including a neurological examination, gave results within normal limits. All laboratory tests including examination of the CSF gave normal results.

On the second day in hospital an ophthalmic plastic surgery opinion was sought. The child continued to

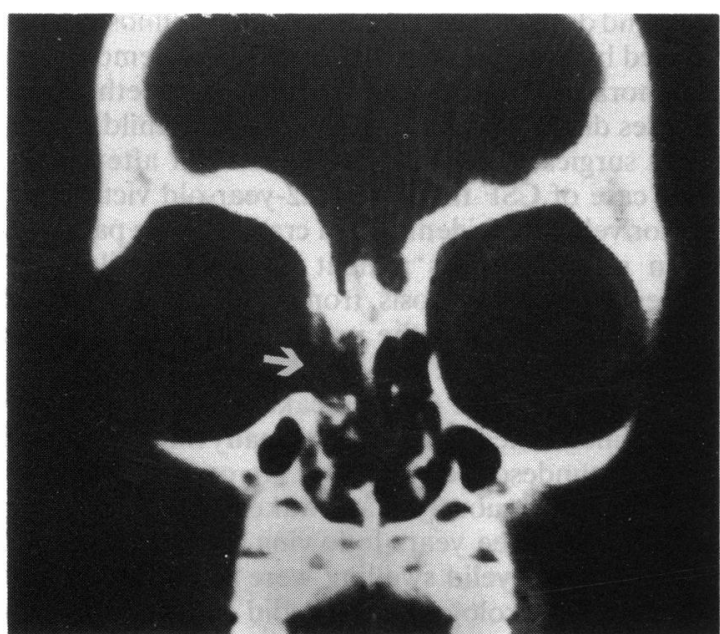

Fig. 1 Coronal CT demonstrating DCR ostium and thickening of nasal mucosa on the right (arrow). 'tear' profusely in the right eye. A Jones primary dye test showed dye in the oropharynx at 2 minutes. It was concluded that the dacryocystorhinostomy surgery had been successful and that causes of pseudoepiphora should be sought to explain the 'tearing'.

Our examination included a Dextrostix test of the tear fluid. The sugar content of the tear fluid was observed. The Dextrostix test of the right eye showed a glucose level of 100 to $180 \mathrm{mg} / \mathrm{dl}(5 \cdot 6-10 \cdot 0 \mathrm{mmol} / \mathrm{l})$. The glucose level from the left eye was less than 100 $\mathrm{mg} / \mathrm{dl}(5.6 \mathrm{mmol} / \mathrm{l})$. A specimen of the patient's tears was collected and sent for laboratory analysis. A discrepancy of 2 to 1 in the glucose concentration existed in the right and left eyes. The data strongly suggested CSF leakage into the orbit. A CT scan was performed (Figs. 1 and 2). A gas lucency in the region of the right orbit with thickening of the right medial rectus and opacification of the right ethmoid was observed. Anteriorly the DCR ostium was visible along with thickening of the nasal mucosa (Fig. 1). A bony defect was noted in the posterior orbit that did not communicate with the DCR ostium (Fig. 2).

On the tenth day in hospital a frontal craniotomy was performed. An encephalocele was found into a fracture in the posterior medial orbital roof. It was retracted from the fracture site, and a Silastic implant was placed over the defect. The dural tear was noted and repaired with a free pericranial graft and closed with a single running suture. Postoperatively the patient did well and was discharged six days later. Tearing ceased immediately after surgery. Measured glucose levels in both eyes were the same and less than $100 \mathrm{mg} / \mathrm{dl}(5.6 \mathrm{mmol} / \mathrm{l})$

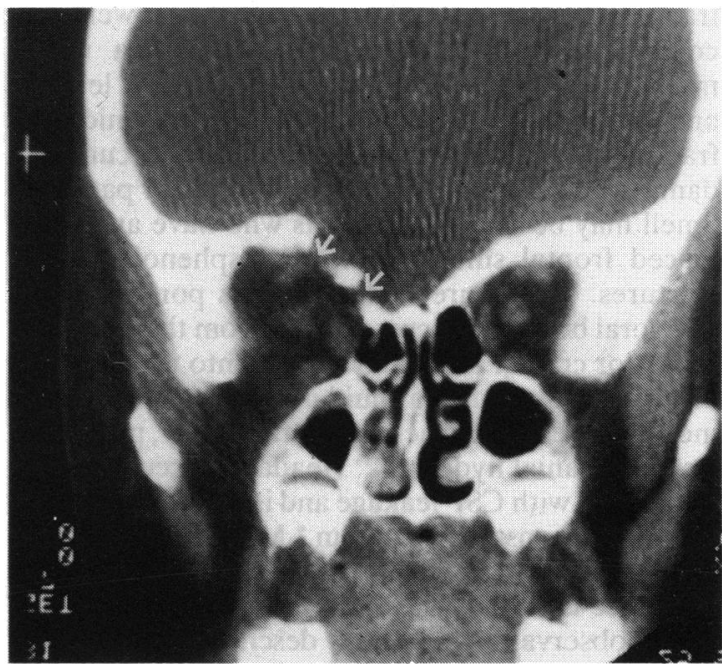

Fig. 2 Coronal CT demonstrating defect in orbital roof on the right (arrows). 


\section{Discussion}

CSF leak has been reported to occur in $6 \%$ to $35 \%$ of patients with severe non-penetrating intracranial injury. ${ }^{159}$ It occurs in approximately $20 \%$ of patients with midfacial fracture or fractures through the paranasal sinuses. ${ }^{59}$

CSF leaks are rare in children below the age of 2 because of the flexibility of the cranial base and the relative immaturity of the paranasal sinuses. However, in children older than 2, CSF leakage is by no means uncommon."

Traumatic CSF leaks usually result from tears in the dura at the skull base that allow CSF to escape from the subarachnoid space into the nasopharynx, the paranasal sinuses, or subcutaneously. The floor of the anterior cranial fossa, particularly the cribriform plate area, is thin, and the dura at this site firmly invests the olfactory fissure where the olfactory nerve penetrates the skull. Midfacial trauma may result in penetration of the anterior cranial fossa by ethmoidal roof or wall fragments and dural tear. CSF escapes immediately. If the edges of the dura are in apposition and do not gape, healing is relatively rapid and CSF leak resolves in three to 10 days. Sinus mucosa may bridge a gaping dural defect and cause leakage to cease. However, if bony spicules project through the tear, a portion of the brain herniates through the defect. If a large dural defect is present, healing may be incomplete. High CSF flow due to coughing or straining may also result in late onset of CSF leakage due to rupture of a partially healed tear. Dural tears may be enlarged by brain and high amplitude CSF pulsation with resultant erosion of bone. ${ }^{9} "$

The diagnosis, aetiology, and management of fractures of the orbital roof have been well described by McClachan et al. ${ }^{12}$ Rhinorrhea is the most common presenting symptom of CSF leakage and presents in $25 \%$ of patients with midfacial fracture. ' Hypo-osmia or anosmia may occur from damage to olfactory nerve fibres in $5 \%$ of patients. ${ }^{9}$ Smell may be intact in patients who have anteriorly placed frontal sinus fractures or sphenoidal sinus fractures. If fracture of the petrous portion of the temporal bone has occurred, CSF from the middle or posterior cranial fossa may escape into the mastoids and produce middle ear fluid mimicking serous otitis media. Rarely CSF tracks subcutaneously, producing a subepicranial hydroma. ${ }^{13}$ Headache occurs in $15 \%$ of patients with CSF leakage and is therefore thought to be an inconstant symptom. ${ }^{9}$ Meningitis occurs in between $3 \%$ and $50 \%$ of cases of CSF leakage and the incidence is higher when the leakage is chronic." 14/16

The observant patient may describe a gush of salty fluid, or choke on arising in the morning. In the recumbent position leaking CSF collects dependently in the sphenoid sinus. On the patient's standing, an influx of CSF collected overnight rushes into the oropharynx causing the so-called reservoir sign. ${ }^{17}$

Other signs of CSF leakage include frequent awakening from sleep with coughing fits and soaking of the bed sheets with clear nasal discharge each night. Rhinitis has also been described from chronic CSF leakage. " 11

CSF leakage following midfacial fracture in an otherwise asymptomatic patient may be confused with traumatic epiphora. In a series of 300 patients with midfacial fracture where $35 \%$ had CSF leakage, $5 \cdot 3 \%$ had concomitant epiphora or dacryocystitis. ${ }^{5}$ In a series of 100 patients reported by Campbell with midfacial fracture $12 \%$ had complaints of tearing. ${ }^{18}$ Naso-orbital trauma may injure the medical palpebral tendon, lacerate or compress the nasolacrimal sac or duct, or cause cicatricial ectropion of the lower lid or punctal malposition. ${ }^{478}$ While CSF leakage usually resolves, dacryocystitis and epiphora commonly persist until surgically treated.

Few cases of chronic CSF leakage into the orbit have been reported in the neurosurgical or ophthalmic literature. In a review of giant epidermoid and dermoid tumors of the orbit Carey described two cases of chronic CSF leakage into the orbit thought to be from direct communication between the subarachnoid space and the inner portion of the tumour. ${ }^{19}$ In his series other symptoms including pulsating proptosis, and extraocular movement limitation were also observed. ${ }^{19}$

A case of traumatic CSF fistula simulating epiphora occurred in a child 8 months of age and was reported in the neurosurgical literature. ${ }^{20}$ Epiphora occurred immediately following head trauma. The patient presented with coma, hemiparesis, and facial weakness and developed a pulsating exophthalmos accompanied by bruit and chronic epiphora. Chemosis and epiphora resulted from a leak through the ethmoidal sinuses directly to the conjunctiva. The child underwent surgical repair of the fistula soon afterwards.

A case of CSF fistula in a 22-year-old victim of a motor vehicle accident with a cranial nerve palsy has been reported. This patient had a pulsatile right upper lid and chemosis from direct subarachnoid communication with the upper lid. No tearing was observed at any time. ${ }^{21}$

The present case of CSF leakage differs from the above-mentioned cases and has many interesting and hitherto undescribed features. It occurred chronically and continuously in an awake and alert child for a period of three years following injury. Proptosis, chemosis, or eyelid swelling were not noted at any time, and neurological deficit did not develop. No pulsations were observed. Finally, tearing was not suspected to be secondary to orbital CSF leakage 
until after successful lacrimal drainage surgery had been performed.

CSF leakage occurred as a result of dacryocystorhinostomy surgery in two cases reported by Neuhaus and Baylis and may occur if bone removal extends to the level of the cribriform plate. ${ }^{22}$ The same authors noted an average of $5 \mathrm{~mm}$ between the nasal ostium and the floor of the anterior cranial fossa..$^{22}$ While it is possible that in the reported cases DCR surgery could have created a CSF leak, it is unlikely. Tearing was chronic prior to surgery and persisted after surgery, and it promptly resolved following neurosurgical repair of the posterior orbital encephalocele. Other unlikely hypotheses that might explain the findings in the presented case include the possibility that CSF leaked from the canaliculi in retrograde fashion from the abnormality of the cranial vault, simulating tearing. The CT evidence for this theory is lacking, as the encephalocele was well posterior to the lacrimal drainage system. While remanipulation of a traumatically altered orbit and cranial base undoubtedly introduced meningeal infection, the pathway of CSF leakage from posterior orbit to the tear film is a matter of speculation.

Reagent strips such as Dextrostix and Labstix commonly used for urine glucose determination, while not helpful in distinguishing CSF from nasal discharge, ${ }^{123-26}$ may be helpful in diagnosing CSF leakage into the orbit. CSF glucose is approximately $70 \%$ of that of serum glucose. Normal levels of glucose in tears are insignificant. ${ }^{2627}$ For the purposes of the clinical examination in the reported case, tear concentration in 20 normal individuals without diabetes mellitus was measured with a Labstix reagent strip by placing it directly on the tear meniscus and waiting until wetting had occurred. After 30 seconds the glucose level was measured by matching the colour of the paper with the colour of the glucose as determined on the table found on the bottle. In all patients the glucose level was found to be within 0 and $100 \mathrm{mg} / \mathrm{dl}(0$ and $5.6 \mathrm{mmol} / \mathrm{l})$. In the case described here the glucose level on the uninvolved side was from 0 to $100 \mathrm{mg} / \mathrm{dl}$, corresponding to the normal value. On the involved side the tear glucose level was between 100 and $180 \mathrm{mg} / \mathrm{dl}(5.6$ and 10.0 $\mathrm{mmol} / \mathrm{l})$. The higher glucose level on the involved side in this patient was confirmed by laboratory analysis. A large sample needs to be collected for glucose concentration to be analysed in a laboratory. The reagent strips are inexpensive, immediate, and require substantially less tear volume and were effective in diagnosing the leakage.

The site of CSF leakage may be localised radiographically with or without the aid of contrast material. Skull films may show an air fluid level in the sinuses. ${ }^{9}$ Materials can be instilled intrathecally to demonstrate leakage of CSF. Methylene blue and fluorescein were at one time injected but have been abandoned owing to numerous complications. ${ }^{1128} 29$ Radioiodide labelled serum albumin and technetium are used to directly visualise CSF leak with cisternography and are diagnostic in $86 \%$ of cases. ${ }^{30-33}$ Metrizamide encephalocisternography with high resolution CT is mandatory in evaluation of the patient with a suspected chronic orbital CSF leak. In our patient CT scans with axial views were obtained at the time of the original trauma and repeatedly thereafter to evaluate brain, orbits, and skull base and were all within normal limits. One week following DCR axial CTs again did not show any abnormality of the skull base or orbital roof. However, coronal CTs with $1.5 \mathrm{~mm}$ cuts clearly show a posterior bony defect and suggested meningoencephalocele and chronic CSF leak. The different perspective offered by directly formed coronal views may be an asset in considering the possibility of chronic CSF leakage into the orbit.

When a CSF leak is noted in the early period post midfacial injury, opinions are varied as to whether direct and immediate reduction of facial fracture or repair of dural tear is indicated. Most authors recommend bed rest with the head of the bed raised. Prophylactic antibiotics are administered intravenously. Bed rest, stool softeners, and medication to prevent coughing or straining are recommended. A lumbar drain, placed in the lumbar subarachnoid space, may be useful in lessening CSF flow through the dural rent, thus allowing repair to take place. Likewise, acetazolamide may be administered to decrease the production of CSF.

The majority of CSF leaks heal without the need for surgery. ${ }^{\text {"I }}$ When the neurological condition has stabilised, fractures may be reduced with the realisation that additional trauma to the dura may result.

The treatment of chronic persistent CSF leak is neurosurgical. 11617 If meningitis has supervened, an adequate period of time should elapse for the patient to be on appropriate antibiotics. Chronic CSF leaks usually do not subside spontaneously. The area of the dural rent must be localised and repaired. In the reported case a frontal craniotomy with exposure of the fracture site, Silastic roof implant, and epicranial duroplasty were performed. The techniques of this neurosurgical procedure go beyond the scope of this paper.

Patients who tear after naso-orbital trauma must be suspected of having a CSF leak even if signs of nasolacrimal duct obstruction are present. It may be useful to ask patients specifically about rhinorrhoea or anosmia, as well as to examine for increased tearing during the Valsalva manoeuvre. CT scans 
should be reviewed if they have been performed and coronal sections are preferred. Finally, Dextrostix can be used to obtain an objective measurement of tear sugar level in order to diagnose chronic orbital CSF leak. If CSF leak is a possibility, the patient should be referred for neurosurgical consultation prior to any lacrimal surgical intervention.

The authors are grateful to Jack H Dunn, the neurosurgeon involved in this case.

\section{References}

1 Dawson RLG, Fordyce GL. Complex fractures of the middle third of the face and their early treatment. BrJ Surg 1953; 41: 254-68.

2 Converse JM, Smith B. Naso-orbital fractures. Ophthalmology (Rochester) 1963; 67: 622-34.

3 Converse JM, Smith B. Malunited fractures of the bones of the orbit. In: Converse JM, ed. Reconstructive plastic surgery; principles and procedures in correction, reconstruction, and transplantation. Philadelphia: Saunders, 1964; 2: 645-61.

4 Converse JM, Smith B. Naso-orbital fractures and traumatic deformities of the medial canthus. Plast Reconstr Surg 1966; 38: $147-62$.

5 Morgan BDG, Madan DK, Bergerot JPC. Fractures of the middle third of the face-a review of 300 cases. Br J Plast Surg 1972; 25: 147-51.

6 Beyer CK, Smith B. Naso-orbital fractures: their complications and treatment. In: Tessier P, Callahan A, Mustarde JC, Salyer $\mathrm{KE}$, eds. Symposium on plastic surgery in the orbital region. St Louis: Mosby, 1976: 107-12.

7 Converse JM. Orbital and naso-orbital fractures. In: Tessier P, Callahan A, Mustarde JC, Salyer KE, eds. Symposium on plastic surgery in the orbital region. St Louis: Mosby, 1976: 79-106.

8 Beyer CK, Fabian RL, Smith B. Naso-orbital fractures, complications, and treatment. Ophthalmology (Rochester) 1982; 89: 456-63.

9 Lewin W. Cerebrospinal fluid rhinorrhea in closed head injuries. Br J Surg 1954; 42: 1-18.

10 Caldicott WJH, North JB, Simpson DA. Traumatic cerebrospinal fluid fistula in children. J Neurosurg 1973; 38: 1-9.

11 Spetzler RJ, Wilson CB. Dural fistulae and their repair. In: Youmans JR, ed. Neurological surgery. 2nd ed. Philadelphia: Saunders, 1982; 4: 2209-27.

12 McClachan DL, Flanagan JC, Shannon GN. Complications of orbital roof fractures. Ophthalmology (Rochester) 1982; 89: 1274-8.

13 Cullen JR. Unfamiliar swelling after head trauma. Clin Pediatr 1974; 13: 378 .
14 Brawley B, Kelly W. Treatment of skull fractures with and without cerebrospinal fluid fistula. J Neurosurg 1967; 26: 57-61.

15 Mincy JE. Post-traumatic cerebrospinal fluid fistula of the frontal fossa. J Trauma 1966; 6: 618-22.

16 Raskind R, Doria A. Cerebrospinal fluid rhinorrhea and otorrhea of traumatic origin. Int Surg 1966; 46: 223-6.

17 Dandy WE. Treatment of rhinorrhea and otorrhea. Arch Surg 1944; 49: 75-85.

18 Campbell $\mathrm{W}$. The radiology of the lacrimal system. Br J Radiol 1964; 37: 1-26.

19 Carey PC. Epidermoid and dermoid tumors of the orbit. Br J Ophthalmol 1958; 42: 225-39.

20 Joshi KK, Crockard A. Traumatic cerebrospinal fluid fistula simulating tears. J Neurosurg 1978; 49: 121-3.

21 Galzio RJ, Lucantoni D, Zenobii M, et al. Traumatic craniopalpebral cerebrospinal fluid fistula. J Neurosurg Sci 1981; 25: 105-7.

22 Neuhaus RW, Baylis HI. Cerebrospinal fluid leakage after dacryocystorhinostomy. Ophthalmology (Rochester) 1983; 90: 1091-5.

23 Dadeholt $\mathrm{H}$. The reaction of glucose oxidase test paper in normal nasal secretion. Acta Otolaryngol 1964; 58: 271-2.

24 Kirsch AP. Diagnosis of cerebrospinal fluid rhinorrhea: lack of specificity of the glucose oxidase test tape. $J$ Pediatr 1967; 71: 718-9.

25 Healy CE. The significance of a positive reaction for glucose in rhinorrhea. Clin Pediatr 1969; 8: 239.

26 Van Haeringen JH. Clinical biochemistry of tears. In: Milder B, Weil B, eds. The lacrimal system. Norwalk, CT: AppletonCentury Crofts, 1983: 23-48.

27 Gasset AR, Braverman LE, Fleming MC, McArky RA, Alter BR. Tear glucose detection of hyperglycemia. Am J Ophthalmol 1968; 65: 414-20.

28 Evans JP, Keegan HR. Danger in the use of intrathecal methylene blue. JAMA 1960; 174: 856-9.

29 Wallace JD, Weintraub MI, Mattson RH, Rosenagle R. Status epilepticus as a complication of intrathecal fluorescein, a case report. J Neurosurg 1972; 36: 659-70.

30 DiChiro G, Reames PM, Matthews WB. RISA ventriculography and RISA cisternography. Neurology (NY) 1964; 14: 185-91.

31 Allen MB, Jr, Gammal TE, Ihnen M, Cowan MA. Fistula detection in cerebrospinal fluid leakage. J Neurol Neurosurg Psychiatry 1972; 35: 664-8.

32 Ashburn WL, Harbert JC, Briner WH, DiChiro G. Cerebrospinal fluid rhinorrhea study with a gamma camera. J Nucl Med 1968; 9: 523-9.

33 DiChiro G, Ommaya AK, Ashburn WL, Briner WH. Isotope cisternography in the diagnosis and follow-up of cerebrospinal fluid rhinorrhea. J Neurosurg 1968; 28: 522-9.

Accepted for publication 3 December 1985. 